\title{
Constitutive equation for friction with rotational and orthotropic anisotropy
}

\author{
K. Hashiguchi* and S. Ozaki** \\ * Member Dr. of Eng. and Dr. of Agr., Prof., Dept. Civil and Enveron. Eng., \\ College of Technology, Daiichi University, \\ (Kokubu-Chuo 1-10-2, Kirishima-shi, Kagoshima-ken 899-4395, Japan) \\ ** Dr. of Agr., Research Associate, Department of Mechanical Engineering, \\ Tokyo University of Science (Kudankita 1-14-6, Chiyoda-ku, Tokyo 102-0073, Japan)
}

\begin{abstract}
The constitutive equation of friction has been developed by the authors so as to describe the smooth transition from the elastic to plastic sliding state and the transition from the static to the kinetic friction and its vice versa, which is called the time-dependent subloading-friction model. In this article this model is further extended so as to describe the anisotropic hardening by incorporating the concept of the rotation of yield surface, i.e. the rotational hardening that has been adopted in the elastoplastic constitutive equation of soils.
\end{abstract}

Key Words: Anisotropy, constitutive law, friction, rotational hardening, subloading surface model, time-dependence

\section{Introduction}

Description of the friction phenomenon as constitutive equations has been attained first as a rigid-plasticity ${ }^{1,2)}$. Further, they have been extended to an elastoplasticity ${ }^{3 / 16}$ in which the penalty concept, i.e. the fictitious springs between contact surfaces is incorporated and the isotropic hardening is taken into account so as to describe the test results ${ }^{17)}$ exhibiting the smooth contact traction vs. sliding displacement curve reaching the static-friction. However, the interior of the sliding-yield surface has been assumed to be an elastic domain and thus the plastic-sliding velocity due to the rate of traction inside the sliding-yield surface and the accumulation of plastic-sliding due to the cyclic loading of contact traction cannot be described by these models. They could be called the conventional friction model in accordance with the classification of plastic constitutive models by Drucker ${ }^{18)}$. On the other hand, the first author of the present article has proposed the subloading surface model ${ }^{19)}$ ${ }^{-21)}$ within the framework of unconventional plasticity, which is capable of describing the plastic strain rate by the rate of stress inside the yield surface. Further, the authors proposed the subloading-friction mode $l^{22}$ describing the smooth transition from the elastic- to the plastic-sliding state by incorporating the concept of subloading surface. Besides, in this model the de- crease of friction coefficient with the increase of normal contact traction observed in experiments ${ }^{23,24)}$ is described by incorporating the nonlinear sliding-yield surface, while the decrease has not been taken into account in Coulomb friction law which has been adopted widely in friction models.

It is widely known that when bodies at rest begin to slide to each other, a high friction coefficient appears first, which is called the static-friction, and then it decreases approaching a stationary value, called the kinetic-friction. Further, it has been found that if the sliding ceases for a while and then it starts again, the static-friction recovers and the identical behavior as that in the initial sliding is reproduced ${ }^{24)-36}$. The recovery has been formulated by equations ${ }^{24)-36}$ including the time elapsed after the stop of sliding. However, the inclusion of time itself leads to the loss of objectivity in constitutive equations as known from the fact that the elapsed time varies depending on the judgment of time as the stop of sliding, which is accompanied with the arbitrariness especially when the sliding velocity fluctuates. Here, it should be noted that the variation of material property has to be described by internal variables and their rates.

The decrease of friction coefficient from the static- to kinetic-friction and the recovery of friction coefficient mentioned above are to be the fundamental behavior of friction between bodies, which have been recognized widely. Difference of the 
static- and the kinetic-friction often reaches up to several tens percents. Therefore, the formulation of the transition from the static- to the kinetic-friction and vice versa is of importance for the development of mechanical design in the field of engineering. Then, the subloading-friction model ${ }^{22)}$ is extended so as to describe the decrease of friction coefficient from the static- to kinetic-friction by the softening due to the plastic-sliding and the recovery of friction coefficient by the hardening due to the creep deformation under the contact pressure in the recent paper $^{37)}$, whilst it is called the time-dependent subloading-friction model. However, it has been limited to the isotropic hardening/softening so far.

In this article the time-dependent subloading-friction model is extended so as to describe the anisotropic hardening by incorporating the concept of the rotation of yield surface, i.e. the rotational hardening ${ }^{39,}$ 44)-46) which has been adopted in the elastoplastic constitutive equation of soils.

\section{Decomposition of sliding velocity}

The sliding velocity $\overline{\mathbf{v}}$ between contact surfaces is additively decomposed into the normal component $\overline{\mathbf{v}}_{n}$ and the tangential component $\overline{\mathbf{v}}_{t}$ as follows:

which are given by

$$
\overline{\mathbf{v}}=\overline{\mathbf{v}}_{n}+\overline{\mathbf{v}}_{t},
$$

$$
\left.\begin{array}{l}
\overline{\mathbf{v}}_{n}=(\overline{\mathbf{v}} \bullet \mathbf{n}) \mathbf{n}=(\mathbf{n} \otimes \mathbf{n}) \overline{\mathbf{v}}, \\
\overline{\mathbf{v}}_{t}=\overline{\mathbf{v}}-\overline{\mathbf{v}}_{n}=(\mathbf{I}-\mathbf{n} \otimes \mathbf{n}) \overline{\mathbf{v}},
\end{array}\right\}
$$

where $\mathbf{n}$ is the unit outward-normal vector at the contact surface, $(\bullet)$ and $\otimes$ denote the scalar and the tensor products, respectively, and I is the second-order identity tensor having the components of Kronecker's delta $\delta_{i j}=1$ for $i=j$, $\delta_{i j}=0$ for $i \neq j$. On the other hand, it is assumed that $\overline{\mathbf{v}}$ is additively decomposed into the elastic -sliding velocity $\overline{\mathbf{v}}^{e}$ and the plastic-sliding velocity $\overline{\mathbf{v}}^{p}$, i.e.

with

$$
\overline{\mathbf{v}}=\overline{\mathbf{v}}^{e}+\overline{\mathbf{v}}^{p}
$$

$$
\begin{aligned}
& \overline{\mathbf{v}}_{n}=\overline{\mathbf{v}}_{n}^{e}+\overline{\mathbf{v}}_{n}^{p}, \\
& \overline{\mathbf{v}}_{t}=\overline{\mathbf{v}}_{t}^{e}+\overline{\mathbf{v}}_{t}^{p} \text {. } \\
& \left.\left.\begin{array}{l}
\overline{\mathbf{v}}_{n}^{e}=\left(\overline{\mathbf{v}}^{e} \bullet \mathbf{n}\right) \mathbf{n} \\
\overline{\mathbf{v}}_{t}^{e}=\overline{\mathbf{v}}-\overline{\mathbf{v}}_{n}^{e}
\end{array}\right\} \begin{array}{l}
\overline{\mathbf{v}}_{n}^{p}=\left(\overline{\mathbf{v}}^{p} \bullet \mathbf{n}\right) \mathbf{n} \\
\overline{\mathbf{v}}_{t}^{p}=\overline{\mathbf{v}}-\overline{\mathbf{v}}_{n}^{p}
\end{array}\right\}
\end{aligned}
$$

First, let the elastic-sliding velocity be given by

$$
\overline{\mathbf{v}}_{n}^{e}=-\frac{1}{\alpha_{n}} \stackrel{\circ}{n}_{n}, \quad \overline{\mathbf{v}}_{t}^{e}=-\frac{1}{\alpha_{t}} \stackrel{\circ}{t}_{t}
$$

where $\mathbf{f}_{n}$ and $\mathbf{f}_{t}$ are the normal component and tangential component, respectively, of the traction vector $\mathbf{f}$ applied to a unit area of contact surface, i.e.

$$
\left.\begin{array}{l}
\mathbf{f}_{n} \equiv(\mathbf{n} \cdot \mathbf{f}) \mathbf{n}=(\mathbf{n} \otimes \mathbf{n}) \mathbf{f} \\
\mathbf{f}_{t} \equiv \mathbf{f}-\mathbf{f}_{n}=(\mathbf{I}-\mathbf{n} \otimes \mathbf{n}) \mathbf{f}
\end{array}\right\}
$$

and, $\left(^{\circ}\right)$ denoting the corotational rate, $\stackrel{\circ}{\mathbf{f}}_{n}$ and $\stackrel{\circ}{f}_{t}$ are the normal component and tangential component, respectively, of the corotational rate $\mathbf{f}$ of the traction vector $\mathbf{f}$, which are related to the material-time derivative denoted by $\left(^{\bullet}\right)$ as follows:

$$
\stackrel{\circ}{\mathbf{f}}=\dot{\mathbf{f}}-\mathbf{\Omega f}, \quad \stackrel{\circ}{\mathbf{f}_{n}}=\dot{\mathbf{f}}_{n}-\mathbf{\Omega} \mathbf{f}_{n}, \quad \stackrel{\circ}{\mathbf{f}_{t}}=\dot{\mathbf{f}}_{t}-\mathbf{\Omega} \mathbf{f}_{t}
$$

where the skew-symmetric tensor $\boldsymbol{\Omega}$ designates the rigid-body rotation of the contact surface. $\alpha_{n}$ and $\alpha_{t}$ are the contact elastic moduli in the normal and the tangential directions to the contact surface. Thus, it follows from Eq. (6) that

$$
\stackrel{\circ}{\mathbf{f}}=\stackrel{\circ}{\mathbf{f}}_{n}+\stackrel{\circ}{\mathbf{f}}_{t}=-\mathbf{C}^{e} \overline{\mathbf{v}}^{e}
$$

where the second-order tensor $\mathbf{C}^{e}$ is the fictitious contact elastic modulus tensor between contact surfaces and is decomposed into the normal and tangential components, i.e.,

with

$$
\mathbf{C}^{e}=\mathbf{C}_{n}^{e}+\mathbf{C}_{t}^{e}
$$

$$
\mathbf{C}_{n}^{e}=\alpha_{n} \mathbf{n} \otimes \mathbf{n}, \mathbf{C}_{t}^{e}=\alpha_{t}(\mathbf{I}-\mathbf{n} \otimes \mathbf{n})
$$

\section{Normat-sliding and sliding-subloading surfaces}

The sliding-yield surface for friction phenomenon would be similar to that of soils having the frictional priperty. Then, assume the following sliding-yield surface with the rotational hardening.

$$
f(\mathbf{f}, \boldsymbol{\beta})=F,
$$

where $F$ is the isotropic hardening/softening function denoting the variation of the size of sliding-yield surface, $\beta$ is the vector $^{39)}$ describing the rotation of sliding-yield surface, called the rotational hardening variable. In what follows, we assume that the interior of the sliding-yield surface is not a purely elastic domain but that the plastic-sliding velocity is induced by the variation of traction inside that surface. Therefore, let the surface described by Eq. (12) be renamed the normal-sliding surface.

Next, in accordance with the concept of subloading surface, we introduce the sliding-subloading surface, which always passes through the current traction $\mathbf{f}$ and keeps a similar shape and a same orientation to the normal-sliding surface with respect to the zero traction point $\mathbf{f}=\mathbf{0}$. Then, the sliding-subloading surface fulfills the following geometrical characteristics.

i ) All lines connecting an arbitrary point inside the sliding-subloading surface and its conjugate point inside the normal-sliding surface join at a unique point, called the similarity-center, which is the ori- 
gin of the contact traction space in the present model.

ii) All ratios of length of an arbitrary line-element connecting two points inside the sliding-subloading surface to that of an arbitrary conjugate line-element connecting two conjugate points inside the normal-sliding surface are identical. The ratio is called the similarity-ratio, which coincides with the ratio of the sizes of these surfaces.

Let the similarity-ratio of the sliding-subloading surface to the normal-sliding surface be called the normal-sliding ratio, denoted by $\bar{R}(0 \leq \bar{R} \leq 1)$, where $\bar{R}=0$ corresponds to the null traction state $(f=0)$ as the most elastic state, $0<\bar{R}<1$ to the subsliding state $(0<f<F)$, and $\bar{R}=1$ to the normal-sliding state in which the traction lies on the normal-sliding surface $(f=F)$. Therefore, the normal-sliding ratio $\bar{R}$ plays the role of three-dimensional measure of the degree of approach to the normal-sliding state. Then, the sliding-subloading surface is described by

$$
f(\mathbf{f}, \boldsymbol{\beta})=\bar{R} F .
$$

The material-time derivative of Eq. (13) leads to

$$
\frac{\partial f}{\partial \mathbf{f}} \bullet \stackrel{\circ}{\mathbf{f}}+\frac{\partial f}{\partial \boldsymbol{\beta}} \bullet \stackrel{\circ}{\boldsymbol{\beta}}=\bar{R} \dot{F}+\stackrel{\bullet}{R} F
$$

The transformation of the material-time derivative of scalar function to the corotational derivative is verified by the first author $^{47)}$.

\section{Evolution rule of hardening function}

It could be stated from experiments that

1 ) If the sliding commences, the friction coefficient reaches first the highest value of static-friction and then it reduces to the lowest stationary value of kinetic-friction. Physically, this phenomenon could be interpreted to be caused by the separation of adhesion of surface asperities between contact bodies due to the plastic-sliding. Then, let it be assumed that the reduction is caused by the contraction of the normal-sliding surface, i.e. the isotropic softening due to the plastic-sliding.

2 ) If the sliding ceases after the reduction of friction coefficient, the friction coefficient recovers gradually with the elapse of time and the static-friction is reproduced after a sufficient time. Physically, this phenomenon could be interpreted to be caused by the reconstruction of the adhesion of surface asperities between contact bodies subjected to the contact pressure. Then, let it be assumed that the recovery is caused by the expansion of the normal-sliding surface, i.e. the isotropic hardening due to the creep deformation under the contact pressure.
Taking account of these facts, let the evolution rule of the isotropic hardening function $F$ be postulated as follows:

$$
\stackrel{\bullet}{F}=-\kappa\left(\frac{F}{F_{k}}-1\right)^{m}\left\|\mathbf{r}^{p}\right\|+\eta\left(1-\frac{F}{F_{s}}\right)^{n}
$$

where $F_{s}$ and $F_{k}\left(F_{s}>F_{k}\right)$ are the ultimate values of $F$ for the static- and the kinetic-friction, respectively. $\kappa$ and $m$ are the material constants influencing the decreasing rate of $F$ with the plastic-sliding, and $\eta$ and $n$ are the material constants influencing the recovering rate of $F$ with the elapse of time, while they would be functions of absolute temperature in general. The first and the second terms in Eq. (15) stand for the deterioration and the formation, respectively, of the adhesion between surface asperities.

\section{Evolution rule of normal-sliding ratio}

It is observed in experiments that the tangential traction increases almost elastically until it reaches to some value and thereafter its increasing rate lowers gradually approaching the normal-sliding surface in the plastic-sliding process. Then, we assume the evolution rule of the normal-sliding ratio as follows:

$$
\stackrel{\bullet}{R}=\bar{U}(\bar{R})\left\|\overline{\mathbf{v}}^{p}\right\| \text { for } \overline{\mathbf{v}}^{p} \neq \mathbf{0}
$$

where $\bar{U}(\bar{R})$ is a monotonically decreasing function of $\bar{R}$ fulfilling the following conditions.

$$
\left.\begin{array}{l}
\bar{U}(\bar{R}) \rightarrow+\infty \text { for } \bar{R} \leq \bar{R}_{e} \\
\bar{U}(\bar{R})=0 \text { for } \bar{R}=1 \\
(\bar{U}(\bar{R})<0 \text { for } \bar{R}>1)
\end{array}\right\}
$$

where $\bar{R}_{e}(<1)$ is the material constant. The simplest function $\bar{U}(\bar{R})$ fulfilling Eq. (17) is given as follows ${ }^{21) .38)}$

$$
\bar{U}(\bar{R})=-\bar{u} \ln \frac{\left\langle\bar{R}-\bar{R}_{e}\right\rangle}{1-\bar{R}_{e}}
$$

where $\bar{u}$ is the material constant. The symbol \langle\rangle is the McCauley's bracket, i.e. $\langle s\rangle=s$ for $s \geq 0$ and $\langle s\rangle=0$ for $s<0$ for arbitrary scalar variable $s$.

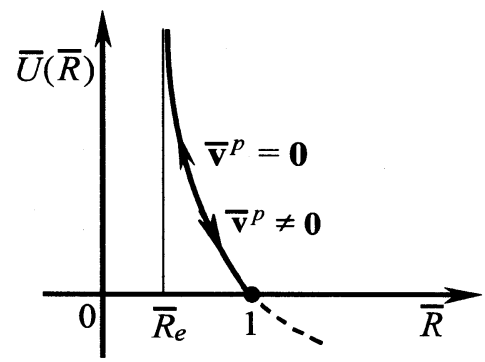

Fig. 1. Function $\bar{U}(\bar{R})$

\section{Sliding-yield surface and rotational hardening rule}

Let it be assumed that the sliding-yield function $f(\mathbf{f}, \boldsymbol{\beta})$ is described by

$$
f(\mathbf{f}, \boldsymbol{\beta})=f_{n} g(\hat{\chi})
$$


where

$$
\hat{\chi} \equiv \frac{\|\hat{\boldsymbol{\eta}}\|}{M}, \hat{\boldsymbol{\eta}} \equiv \boldsymbol{\eta}-\boldsymbol{\beta}, \boldsymbol{\eta} \equiv \frac{\mathbf{f}_{t}}{f_{n}}, f_{n}=\mathbf{n} \bullet \mathbf{f}
$$

$M$ is the material constant denoting the traction ratio $\|\boldsymbol{\eta}\|\left(=\left\|\mathbf{f}_{t}\right\| / f_{n}\right)$ at the maximum point of $\left\|\mathbf{f}_{t}\right\|$ for the case $\beta=0$. $\boldsymbol{\beta}$ is the vector describing the rotation of the sliding-yield surface ${ }^{39)}$ and is called the rotational hardening variable. The simple examples of the function $g(\hat{\chi})$ in the sliding-yield function in Eq. (19) are as follows;

$$
\begin{gathered}
g(\hat{\chi})=\exp (\hat{\chi}) \\
g(\hat{\chi})=1+\hat{\chi}^{2} \\
g(\hat{\chi})=\exp \left(\hat{\chi}^{2} / 2\right) \\
g(\hat{\chi})=\frac{1}{1-\hat{\chi} / 2}
\end{gathered}
$$

All the sets of Eqs. (12) and (19) with Eqs. (21)-(24) exhibit the closed surfaces passing through the points $f_{n}=0$ and $f_{n}=F$ at $\mathbf{f}_{t}=\mathbf{0}$ for the isotropic case $\boldsymbol{\beta}=\mathbf{0}$. Eqs. (21) and (22) are based on the original and modified Cam-clay yield surfaces for solis ${ }^{40)}{ }^{41)}$. Eq. (23) exhibits the tear-shaped surface ${ }^{42), 43)}$ which is reversed from the surface of Eq. (21) with respect to the normal traction. Eq. (24) exhibits the parabola.

In what follows let the evolution rule of $\beta$ be formulated based adopting the following assumptions in accordance with the rotational hardening rule of soils ${ }^{44)-46)}$

1. The rotation of sliding-yield surface is not induced by the normal-plastic sliding velocity but is induced by the tangential-plastic sliding velocity, while the anisotropy is induced by the plastic strain rate.

2. The traction-ratio $\boldsymbol{\eta}$ would increase to unrealistically high level as the plastic sliding proceeds if there does not exist any limitation in the range of rotation of yield surface. Therefore, there would have to exist the limitation in the range of the rotation of sliding-yield surface such that the rotation of the central axis of sliding-yield surface saturates when the central axis reaches the following surface, called the rotational limit surface, as illustrated in Fig. 2.

$$
\|\boldsymbol{\eta}\|=M_{r}=\tan \phi_{r}
$$

where $M_{r}$ and $\phi_{r}$ is the material constants, called the rotational limit angle. Eq. (25) exhibits the conical surface in the traction space.

3. The central axis $\boldsymbol{\eta}=\boldsymbol{\beta}$ of sliding-yield surface rotates towards the conjugate generating line $\boldsymbol{\eta}=M_{r} \hat{\mathbf{t}} \quad\left(\hat{\mathbf{t}} \equiv \hat{\mathbf{f}}_{t} /\left\|\hat{\mathbf{f}}_{t}\right\|\right)$ on the rotational limit surface, while this line is observed in the same direc- tion from the normal traction axis $\boldsymbol{\eta}=\mathbf{0}$ as the direction of the current traction observed from the central axis $\boldsymbol{\eta}=\boldsymbol{\beta}$ of the sliding-yield surface.

4. The central axis of sliding-yield surface is kept to coincide with the traction path in the constant traction process, i.e. the proportional traction process in which the tangential-plastic sliding deformation proceeds. Then, the sliding-yield surface does not rotate in spite of the tangential-plastic sliding in the state that the traction lies on the central axis of sliding-yield surface but the rate of rotation increases as the direction of traction ratio vector deviates from the central axis of sliding-yield surface.

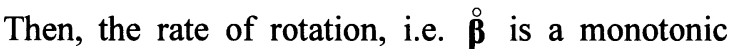
increasing function of $\|\hat{\boldsymbol{\eta}}\|$, whilst the linear function will be adopted in the concrete formulation for simplicity. Besides, this assumption is required also to avoid the discontinuous fluctuation of the direction of rotation in the state that the traction lies on the central axis of sliding-yield surface inducing the tangential-plastic sliding, whilst that point is the singular point in the field of the direction of rotation since the central axis of sliding-yield surface can rotate towards any generating line of the rotational limit surface. The rotated normal sliding, sliding-subloading and rotational limit surfaces for Eq. (22) of ellipsoid are illustrated in Fig. 2.

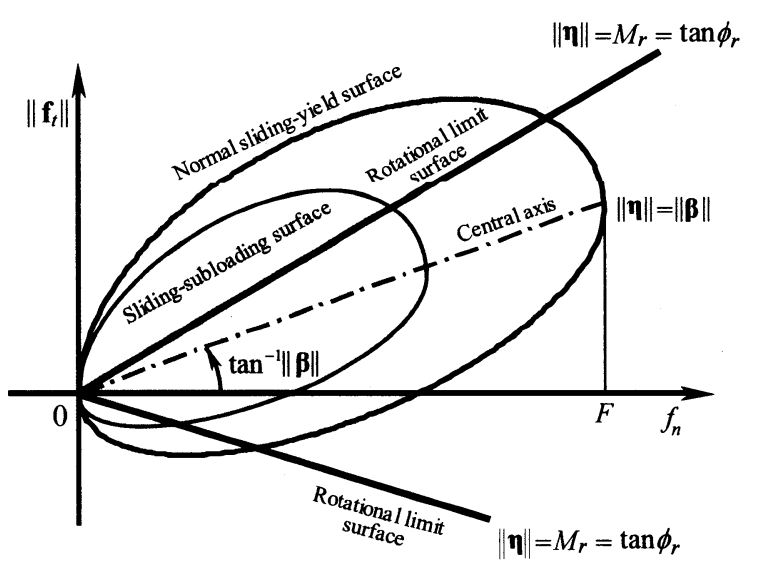

Fig. 2. Rotated normal sliding, sliding-subloading and rotational limit surfaces.

Based on the above-mentioned assumptions let the evolution rule of $\boldsymbol{\beta}$ be given by the following equation which is similar to the rotational hardening rule of soils ${ }^{44)-46)}$.

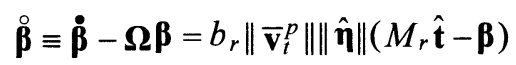

where $b_{r}$ is the material constant.

\section{7. constitutive equation}


The substitution of Eqs. (15), (16) and (26) into Eq. (14) gives rise to the consistency condition for the sliding-subloading surface:

$$
\begin{aligned}
& \frac{\partial f}{\partial \mathbf{f}} \bullet \hat{\mathbf{f}}+b_{r}\left\|\overline{\mathbf{v}}_{t}^{p}\right\|\|\hat{\boldsymbol{\eta}}\| \frac{\partial f}{\partial \boldsymbol{\beta}} \bullet\left(M_{r} \hat{\mathbf{t}}-\boldsymbol{\beta}\right) \\
& \quad=\bar{R}\left\{-\kappa\left(\frac{F}{F_{k}}-1\right)^{m}\left\|\overline{\mathbf{v}}^{p}\right\|+\eta\left(1-\frac{F}{F_{S}}\right)^{n}\right\}+\bar{U}\left\|\overline{\mathbf{v}}_{t}^{p}\right\| F
\end{aligned}
$$

Assume the following sliding-plastic flow rule.

$$
\overline{\mathbf{v}}^{p}=-\bar{\lambda} \mathbf{m}(\bar{\lambda}>0)
$$

where $\bar{\lambda}$ is a positive proportionality factor, where the sign minus in the right-hand side is added since the plastic-sliding rate is induced in the opposite direction of the tangential traction. Substituting Eq. (28) into Eq. (27), the proportionality factor $\bar{\lambda}$ is derived as follows:

$$
\bar{\lambda}=\frac{-\mathbf{N} \cdot \stackrel{\circ}{\mathbf{f}}+M^{c}}{M^{f}}
$$

and thus

$$
\overline{\mathbf{v}}^{p}=-\frac{-\mathbf{N} \cdot \stackrel{\circ}{\mathbf{f}}+M^{c}}{M^{f}} \mathbf{m}
$$

where

$$
\begin{gathered}
M^{c} \equiv \eta \frac{1}{F}\left(1-\frac{F}{F_{s}}\right)^{n} \mathbf{N} \cdot \mathbf{f}(\geq 0) \\
M^{f} \equiv\left\{\frac{\kappa}{F}\left(\frac{F}{F_{k}}-1\right)^{m}-\frac{\bar{U}}{\bar{R}}\right. \\
\left.+\frac{b_{r}}{\bar{R} F}\|\mathbf{m}-(\mathbf{n} \cdot \mathbf{m}) \mathbf{n}\|\|\hat{\mathbf{\eta}}\| \frac{\partial f}{\partial \boldsymbol{\beta}} \bullet\left(M_{r} \hat{\mathbf{t}}-\boldsymbol{\beta}\right)\right\} \mathbf{N} \cdot \mathbf{f}
\end{gathered}
$$

Substituting Eqs. (9) and (30) into Eq. (3), the sliding velocity is given by

$$
\overline{\mathbf{v}}=-\mathbf{C}^{e^{-1}} \stackrel{\circ}{\mathbf{f}}-\frac{-\mathbf{N} \cdot \stackrel{\circ}{\mathbf{f}}+M^{c}}{M^{f}} \mathbf{m}
$$

The positive proportionality factor in terms of the sliding velocity, denoted by the symbol $\bar{\Lambda}$, is given from Eqs. (33) as

$$
\bar{\Lambda}=\frac{-\mathbf{N} \cdot \mathbf{C}^{e} \cdot \overline{\mathbf{v}}-M^{c}}{-M^{f}+\mathbf{N} \cdot \mathbf{C}^{e} \cdot \mathbf{m}}
$$

The traction rate is derived from Fqs. (3), (9) and (28) as follows:

$$
\stackrel{\circ}{\mathbf{f}}=-\mathbf{C}^{e}\left(\overline{\mathbf{v}}-\left\langle\frac{-\mathbf{N} \cdot \mathbf{C}^{e} \cdot \overline{\mathbf{v}}-M^{c}}{-M^{f}+\mathbf{N} \cdot \mathbf{C}^{e} \cdot \mathbf{m}}\right\rangle \mathbf{m}\right)
$$

The loading criterion for the plastic-sliding velocity is given as follows:

$$
\left.\begin{array}{l}
\overline{\mathbf{v}}^{p} \neq \mathbf{0}: \bar{\Lambda}>0 \\
\overline{\mathbf{v}}^{p}=\mathbf{0}: \text { otherwise, }
\end{array}\right\}
$$

due to the requirement of positiveness for the proportionality factor $\bar{\Lambda}$.

By setting

$$
F=f_{n}, M=\mu, g(\hat{\chi})=\hat{\chi}
$$

in the equations formulated in this section the constitutive equation for the Coulomb friction condition ( $\mu$ : coefficient of friction) with the rotational anisotropy is obtained, where the normal-sliding and sliding-subloading surfaces are given as

$$
\left\|\mathbf{f}_{t}-f_{n} \boldsymbol{\beta}\right\|=\mu f_{n},\left\|\mathbf{f}_{t}-f_{n} \boldsymbol{\beta}\right\|=\bar{R} \mu f_{n}
$$

\section{Incorporation of orthotropic anisotropy}

The anisotropy due to the rotation of sliding-yield surface was adopted in the present friction model formulated above Let the orthotropic anisotropy proposed by Mroz at al. ${ }^{48), 49)}$ be further incorporated below into the present friction model. Then, adopt the coordinate system $\left(\mathbf{e}_{1}^{*}, \mathbf{e}_{2}^{*}, \mathbf{n}\right)$, where $\mathbf{e}_{1}^{*}$ and $\mathbf{e}_{2}^{*}$ are the unit vectors tangential to the contact surface and are directed towards the maximum and the minimum principal axes, respectively, of orthotropic anisotropy, setting $\mathbf{e}_{3}^{*}=\mathbf{n}$. Then, it can be written as

$$
\left.\begin{array}{l}
\mathbf{f}=f_{t_{1}}^{*} \mathbf{e}_{1}^{*}+f_{t_{2}}^{*} \mathbf{e}_{2}^{*}+f_{n} \mathbf{e}_{3}^{*} \\
\boldsymbol{\beta}=\beta_{t 1}^{*} \mathbf{e}_{1}^{*}+\beta_{t 2}^{*} \mathbf{e}_{2}^{*}+\beta_{n} \mathbf{e}_{3}^{*}
\end{array}\right\}
$$

Then, the normal-sliding and the sliding-subloading surfaces in Eqs. (12) and (13) with Eq. (19) be extended as

$$
f_{n} g\left(\hat{\chi}^{*}\right)=F, \quad f_{n} g\left(\hat{\chi}^{*}\right)=\bar{R} F
$$

where

$$
\begin{gathered}
\hat{\chi}^{*} \equiv \sqrt{\left(\frac{\hat{f}_{t_{1}}^{*}}{M_{1} f_{n}}\right)^{2}+\left(\frac{\hat{f}_{t 2}^{*}}{M_{2} f_{n}}\right)^{2}} \\
\hat{f}_{t_{1}}^{*} \equiv f_{t_{1}}^{*}-f_{n} \beta_{1}^{*}, \hat{f}_{t_{2}}^{*} \equiv f_{t_{2}}^{*}-f_{n} \beta_{2}^{*}
\end{gathered}
$$

$M_{1}$ and $M_{2}$ are the material constants describing the values of $M$ in the directions of the maximum and the minimum anisotropic axes.

Eq. (40) reduces to the Coulomb friction law extended to the rotational and orthotropic anisotropy

$$
\sqrt{\left(\frac{\hat{f}_{t_{1}}^{*}}{\mu_{1}}\right)^{2}+\left(\frac{\hat{f}_{t_{2}}^{*}}{\mu_{2}}\right)^{2}}=f_{n}, \sqrt{\left(\frac{\hat{f}_{t_{1}}^{*}}{\mu_{1}}\right)^{2}+\left(\frac{\hat{f}_{t_{2}}^{*}}{\mu_{2}}\right)^{2}}=\bar{R} f_{n}
$$

by setting

$$
M_{1}=\mu_{1}, M_{2}=\mu_{2}, \quad F=f_{n}, \quad g\left(\hat{\chi}^{*}\right)=\hat{\chi}^{*}
$$

The constitutive equations for the sliding conditions (37), (40) and (43) are derived by substituting them into the equations in section 7.

For the orthotropic material the formulation and the calculation of all quantities have to be done in the coordinate system directed towards the axes of orthotropic anisotropy.

\section{References}


1) Seguchi, Y., Shindo, A., Tomita, Y. and Sunohara, M. (1974): Sliding rule of friction in plastic forming of metal, Compt. Meth. Nonlinear Mech., University of Texas at Austin, pp. 683-692.

2) Fredriksson, B. (1976): Finite element solution of surface nonlinearities in structural mechanics with special emphasis to contact and fracture mechanics problems, Comput. Struct., 6, 281-290.

3) Michalowski, R. and Mroz, Z. (1978): Associated and non-associated sliding rules in contact friction problems, Archiv. Mech., 30, 259-276.

4) Curnier, A. (1984): A theory of friction, Int. J. Solids Struct., 20, 637-647.

5) Oden, J.T. and Pires, E.B. (1983a): Algorithms and numerical results for finite element approximations of contact problems with non-classical friction laws, Computer and Structures, 19, 137-147.

6) Oden, J.T. and Pires, E.B. (1983b): Nonlocal and nonlinear friction laws and variational principles for contact problems in elasticity, J. Appl. Mech. (ASME), 50, 67-76.

7) Cheng, J.-H. and Kikuchi, N. (1985): An incremental constitutive relation of uniaxial contact friction for large deformation analysis, J. Appl. Mech. (ASME), 52, 639-648.

8) Oden, J.T. and Martines, J.A.C (1986): Models and computational methods for dynamic friction phenomena, Computer Methods in Applied Mechanics and Engineering, 52, 527-634.

9) Kikuchi, N. and Oden, J.T. (1988): Contact problem in elasticity: A study of variational inequalities and finite element methods, SIAM, Philadelphia.

10) Wriggers, P., Vu Van, T., and Stein, E. (1990): Finite element formulation of large deformation impact-contact problems with friction, Comput. Struct., 37, 319-331.

11) Peric, D. and Owen, R.J. (1992): Computational model for 3-D contact problems with friction based on the penalty method, Int. J. Numer. Meth. Eng., 35, 1289-1309.

12) Anand, L. (1993): A constitutive model for interface friction, Computational Mechanics, 12 , 197-213.

13) Mroz, Z. and Stupkiewicz, S. (1998): Constitutive model of adhesive and ploughing friction in metal-formimg processes, Int. J. Mech. Sci., 40, 281-303.

14) Gearing, B.P., Moon, H.S. and Anand, L. (2001): A plasticity model for interface friction: application to sheet metal forming, Int. J. Plasticity, 17, 237-271.

15) Laulsen, T.A. (2001): Computational contact and impact mechanics: Fundamentals of modeling in- ternational phenomena in nonlinear finite element analysis, Springer.

16) Wriggers, P. (2003): Computational contact mechanics, J. Wiley, Chichester.

17) Courtney-Pratt, J.S. and Eisner, E. (1957): The effect of a tangential force on the contact metallic bodies, Proc. Roy. Soc. A, 238, 529-550.

18) Drucker, D.C. (1988): Conventional and unconventional plastic response and representation, Appl. Mech. Rev. (ASME), 41, 151-167.

19) Hashiguchi, K. (1978): Plastic constitutive equations of granular materials, Proc. US-Japan Seminar Continuum Mech. Stast. Appr. Mech. Granular Materials, Sendai, pp. 321-329.

20) Hashiguchi, K. (1980): Constitutive equations of elastoplastic materials with elastic-plastic transition, J. Appl. Mech. (ASME), 47, 266-272.

21) Hashiguchi, K. (1989): Subloading surface model in unconventional plasticity, Int. J. Solids Struct., 25, 917-945.

22) Hashiguchi, K., Ozaki, S. and Okayasu, T. (2005): Unconventional friction theory based on the subloading surface concept, Int. J. Solids Struct., 42, 1705-1727.

23) Bay, N. and Wanheim, T. (1976): Real area of contact and friction stresses at high pressure sliding contact. Wear, 38, 201-209.

24) Dunkin, J.E. and Kim, D.E. (1996): Measurement of static friction coefficient between flat surfaces, Wear, 193, 186-192.

25) Dokos, S.J. (1946): Sliding friction under extreme pressure-I, Trans. ASME, 68, A148-156.

26) Rabinowicz, E. (1951): The nature of the static and kinetic coefficients of friction, J. Appl. Phys., 22, 1373-1379.

27) Rabinowicz, E. (1958): The intrinsic variables affecting the stick-slip process, Proc. Phys. Soc., 71, 668-675.

28) Rabinowicz, E. (1965): Friction and Wear of Materials, John Wiley \& Sons, New York.

29) Howe, P.G., Benson, D.P. and Puddington, I.E. (1955): London-Van der Waals' attractive forces between glass surfaces, Can. J. Chem., 33, 1375-1383.

30) Derjaguin, B.V., Push, V.E. and Tolstoi, D.M. (1957): A theory of stick-sliping of solids, Proc. Conf. Lubr. and Wear, Inst. Mech. Eng., London, pp.257-268.

31) Brockley, C.A. and Davis, H.R. (1968): The time-dependence of static friction, J. Lubr. Tech. (ASME). 90, 35-41.

32) Kato, S., Sato, N. and Matsubayashi, T. (1972): Some considerations on characteristics of static friction of machine tool sideway, J. Lubr. Tech. (ASME), 94, 234-247.

33) Richardson, R.S.H. and Noll, H. (1976): Surface friction under time-depemdent loads, Wear, 37, 87-101.

34) Horowitz, F. and Ruina, A. (1989): Slip patterns in a spa- 
tially homogeneous fault model, J. Geophysical Research, 94, pp. 10279-10298.

35) Ferrero, J.F. and Barrau, J.J. (1997): Study of dry friction under small displacements and near-zero sliding velocity, Wear, 209, 322-327.

36) Bureau, L., Baumberger, T, Caroli, C. and Ronsin, O. (2001): Low-velocity friction between macroscopic solids, C. $R$ Acad Sci. Paris, Series IV, Different faces of Tribology, 2, pp. 699-707.

37) Hashiguchi, K. and Ozaki, S. (2007): Generalized friction model with transition of static-kinetic frictions: Theory, Proc. Int. Conf. Comput. Exper. Eng. Sci, pp. 1447-1452.

38) Tsutsumi, S., Toyosada, M. and Hashiguchi, K. (2006): Extended subloading surface model incorporating elastic limit concept, Proc. Plasticity '06, Halifax, pp. pp. 217-219.

39) Sekiguchi, H. and Ohta, H. (1977): Induced anisotropy and its time dependence in clays, Constitutive Equations of Soils (Proc. Spec. Session 9, 9th ICSFME), Tokyo, pp. 229-238.

40) Schofield, A. N., Wroth, C. P. (1968): Critical State Soil Mechanics, McGraw-Hill, London.

41) Roscoe, K. H., Burland, J. B. (1968): On the generalized stress-strain behavior of 'wet' clay, Engineering Plasticity (eds. Heyman, J. and Leckie, F. A.), Cambridge Univ. Press, pp. 535-608.

42) Hashiguchi, K. (1972): On the yielding of frictional plastic body - hardening rule, Proc. 27th Anmual Meeting, JSCE, pp. 105-108.
43) Hashiguchi, K. (1985): Macrometric approaches -staticintrinsically time-independent, Constitutive Laws of Soils (Proc. 11th Int. Conf. Soil Mech. Found. Eng., Disc. Sess. $I A)$, San Francisco, pp. 25-65.

44) Hashiguchi, K. (1994): Subloading surface model with rotational hardening for soils, Proc. Int. Conf. Compt. Meth. Struct. Geotech. Eng., Hong Kong, pp. 807-812.

45) Hashiguchi, K. and Chen, Z.-P (1998): Elastoplastic constitutive equations of soils with the subloading surface and the rotational hardening, Int. J. Numer. Anal. Meth. Geomech., 22, 197-227.

46) Hashiguchi, K. (2001): Description of inherent/induced anisotropy of soils: Rotational hardening rule with objectivity, Soils \& Foundations, 41(6), 139-145.

47) Hashiguchi, K. (2007): General corotational rate tensor and replacement of material-time derivative to corotational derivative of yield function, Comput. Model. Eng. Sci., 17(1), 55-62.

48) Mroz, Z. and Stupkiewicz, S. (1994): An anisotropic friction and wear model, Int. J. Solids Struct., 31, 1113-1131.

49) Hjiaj, M., Feng, Z.-Q., de Saxce, G. and Mroz, Z. (2004): On the modeling of complex anisotropic frictional contact laws, Int. J. Eng. Sci., 42, 1013-1034.

(Received: April 12, 2007) 